\title{
Geographical patterns and predictors of malaria risk in Zambia: Bayesian geostatistical modelling of the 2006 Zambia national malaria indicator survey (ZMIS)
}

Nadine Riedel ${ }^{1}$, Penelope Vounatsou ${ }^{1 *}$, John M Miller², Laura Gosoniu', Elizabeth Chizema-Kawesha ${ }^{3}$, Victor Mukonka ${ }^{3}$, Rick W Steketee ${ }^{2}$

\begin{abstract}
Background: The Zambia Malaria Indicator Survey (ZMIS) of 2006 was the first nation-wide malaria survey, which combined parasitological data with other malaria indicators such as net use, indoor residual spraying and household related aspects. The survey was carried out by the Zambian Ministry of Health and partners with the objective of estimating the coverage of interventions and malaria related burden in children less than five years. In this study, the ZMIS data were analysed in order (i) to estimate an empirical high-resolution parasitological risk map in the country and (ii) to assess the relation between malaria interventions and parasitaemia risk after adjusting for environmental and socio-economic confounders.

Methods: The parasitological risk was predicted from Bayesian geostatistical and spatially independent models relating parasitaemia risk and environmental/climatic predictors of malaria. A number of models were fitted to capture the (potential) non-linearity in the malaria-environment relation and to identify the elapsing time between environmental effects and parasitaemia risk. These models included covariates (a) in categorical scales and (b) in penalized and basis splines terms. Different model validation methods were used to identify the best fitting model. Model-based risk predictions at unobserved locations were obtained via Bayesian predictive distributions for the best fitting model.

Results: Model validation indicated that linear environmental predictors were able to fit the data as well as or even better than more complex non-linear terms and that the data do not support spatial dependence. Overall the averaged population-adjusted parasitaemia risk was $20.0 \%$ in children less than five years with the highest risk predicted in the northern (38.3\%) province. The odds of parasitaemia in children living in a household with at least one bed net decreases by $40 \%$ (Cl: 12\%, 61\%) compared to those without bed nets.

Conclusions: The map of parasitaemia risk together with the prediction error and the population at risk give an important overview of the malaria situation in Zambia. These maps can assist to achieve better resource allocation, health management and to target additional interventions to reduce the burden of malaria in Zambia significantly. Repeated surveys will enable the evaluation of the effectiveness of on-going interventions.
\end{abstract}

\footnotetext{
* Correspondence: penelope.vounatsou@unibas.ch

'Department of Public Health and Epidemiology, Swiss Tropical Institute, PO Box, 4002 Basel, Switzerland
} 


\section{Background}

Malaria is an endemic disease in Zambia with a national incidence of 412 per 1,000 inhabitants in 2006. Despite a drop of the reported cases over the last years, it is still the leading cause of morbidity and mortality accounting for $45 \%$ of hospitalizations and outpatient department visits with 6,000-8,000 reported deaths [1]. Through the National Malaria Strategic Plan (NMSP) 2005-2010, the Ministry of Health and a network of partners are working toward scaling up effective malaria control interventions with the goal of substantially reducing malariarelated burden, especially among vulnerable populations, such as children under five years of age [2]. Lead by the Ministry of Health, numerous partners, including the Global Fund, the President's Malaria Initiative (PMI), the World Bank and the Malaria Control and Evaluation Partnership in Africa (MACEPA) provide support for scaling up malaria control prevention and treatment services throughout Zambia. The national malaria control programme advocates malaria control through widespread distribution of insecticide-treated mosquito nets, application of insecticides in homes, preventive treatment for pregnant women and effective treatment of infected persons [3]. As part of the programme, 5.3 million insecticide-treated nets were distributed all over the country in the years 2006 and 2007 and 85\% households of 15 target district have been sprayed [4]. In addition, in $2007,60 \%$ of all pregnant women got malaria prevention drug and all pregnant woman who visited a public clinic received one insecticide-treated net for herself and every under-five child in the same household [5].

The efforts of malaria reduction require comprehensive baseline maps of malaria risk over the whole country. These maps can guide malaria control at areas of highest need, help limited resources to be distributed more efficiently and assist in the evaluation of the progress of all intervention programmes. Earlier maps of malaria risk in Zambia are based on malaria climatic suitability conditions [6,7], however to date there is no empirical malaria map for the country. Although historical survey data have been compiled by the Mapping Malaria risk in Africa (MARA) project, malaria risk estimates based on these data will not reflect the current situation, which is changing due to ongoing interventions.

In 2006, the Ministry of Health, the Central Statistics Office (CSO), MACEPA, and partners conducted the first national Zambia Malaria Indicator Survey (ZMIS). This is a nationally representative household survey in children under five to assess the coverage of key malaria interventions and to measure malaria-related burden [5]. The survey contains geo-referenced parasitological data for each child that can be used to estimate the malaria risk and draw accurate maps of the current malaria situation in Zambia. In addition, the ZMIS collected information on previous interventions at household level like bed nets or indoor residual spraying (IRS) and socio-economic aspects.

In this paper, the ZMIS data of 2006 were analysed and the first contemporary empirical parasitaemia risk map for the country was produced. The MIS data are expected to be correlated in space due to common environmental exposures, which influence transmission similarly in neighbouring areas. The standard statistical methods assume independence of the observations. To take into account spatial correlation, Bayesian geostatistical models [8] were developed to establish the relation between the parasitaemia data and environmental/climatic predictors of the disease. In addition, the corresponding non-spatial models were fitted for comparison purposes. Environmental data were obtained vie remote sensing (RS). Potential non-linearity in the environmentmalaria relation and elapsing time in the effects of environmental predictors on parasite risk were modelled using predictors in categorical scales and fitted by penalized and basis spline (P- and B-splines) functions. Due to large number of model parameters, Bayesian Markov chain Monte Carlo (MCMC) simulation was used for model fit. Model based predictions estimating the risk at unobserved locations were obtained via Bayesian kriging. Parasitaemia risk estimates were linked to population data and the number of children at risk at province level was calculated.

\section{Methods}

The study area and the ZMIS

Zambia is a republic in Southern Africa. Most parts of the country are high plateau areas covered with savannas and some rivers, valleys and mountains. The country has a tropical climate with the rainy season occurring during December and April.

The ZMIS was carried out from May to June 2006, shortly after the rainy season. The data were obtained from a nationally representative two-stage cluster sample [5]. At the first stage 120 standard enumeration areas (SEA) were randomly selected among about 17,000 SEAs the country is divided. They are located within 58 out of 72 districts from all 9 provinces in Zambia. Within each SEA, a random sample of 25 households was chosen resulting in a total of 3,000 households. A household and a women's questionnaire were conducted with Personal Digital Assistants (PDA). In addition, blood samples in children under five were collected and analysed for anaemia using Hemocue $\mathrm{Hb}$ 201 and malaria parasites using Paracheck Pf and thick and thin blood smears. Households were geo-located 
using the Global Positioning System (GPS). All data were entered in an ACCESS database.

\section{Socio-economic data}

Socio-economic data were obtained from a household survey carried out during the ZMIS. An asset index was created as a weighted sum of 59 different household assets extracted from 17 relevant questions, which were included in the household survey. The weights were calculated by principle component analysis (PCA) on the asset indicators [9]. Then the household asset index was divided into wealth quintiles to create a socio-economic status analysis variable.

\section{Environmental and population density data}

Environmental predictors were extracted from Remote Sensing (RS) sources at spatial and temporal resolutions shown in Table 1. This data are available for free at high spatial and temporal resolution. To take into account the elapsing time between the climatic suitability for malaria transmission and parasitaemia, the climatic data were gathered for different periods (up to one year) prior to the survey starting from May 2005. Day and night land surface temperature (LST), normalized difference vegetation index (NDVI) and land cover types were downloaded from the Moderate Resolution Imaging Spectroradiometer (MODIS) from the U.S. Geological Survey (USGS) Land Processes Distributed Active Archive Center (LP DAAC) [10]. LST data were extracted as averages over 8-day periods at $1 \mathrm{~km}$ spatial resolution. NDVI was obtained as a 16-day average at a $0.25 \mathrm{~km}$ spatial resolution. Land cover data were available from MODIS for the year 2004 and contained 17 different land cover categories as defined by the International Geosphere-Biosphere Programme (IGBP). They were grouped into five categories, namely wetlands, forests, urban areas, shrublands and others. At each cluster location, the land cover covariate was summarized by the proportion of each land type within a radius of 3 $\mathrm{km}$. During the model fit, the category "others" was dropped from the analysis, to avoid effects of colinearity. Daily rainfall estimates (RFE) were taken from Meteosat 7 satellite images and downloaded from the USGS Famine Early Warning Systems Network (FEWS NET) African Data Dissemination Service (ADDS) [11] at $8 \mathrm{~km}$ spatial resolution.

Altitude data were extracted from an interpolated USGS digital elevation model (DEM) [12] available at a spatial resolution of $1 \mathrm{~km}$. The digital maps for three different kinds of water bodies in Zambia (lakes, rivers and wetlands) and urban/rural regions were acquired from the HealthMapper database [13]. The distance from each location to the nearest water body source was calculated in IDRISI 32 (Clark Labs). Estimates of the number of persons living in an area of 500 by 500 square meters were downloaded from the LandScan ${ }^{\mathrm{m}}$ Global Population Database [14] for the year 2006. The percentage of under-five children out of the total Zambia population (17.3\%) was obtained from the 2006 data of the U.S. Census Bureau International Database [15].

The coordinates of the SEAs were calculated by the average of latitude and longitude over all household locations within the SEA. These coordinates were used to link the environmental and malaria data. For the purpose of predicting parasitaemia risk at the unobserved locations, a grid with cell size of $3 \mathrm{~km}$ by $3 \mathrm{~km}$ was overlaid on the Zambia map (resulting in around 100,000 grid cells) and the remote sensing data were also extracted for the centroids of the grid cells.

The MODIS Reprojection Tool (USGS) was used to convert the RS data to geo-referenced maps. Further processing of the environmental data and distance calculation for the water bodies was carried out in IDRISI 32. ArcMap v. 9.1 (ESRI) was used as a mapping tool. Additional data processing was performed in Stata/SE 9.2 (StataCorp LP).

\section{Statistical models}

Most of the climatic RS data are available continuously in time. Depending on the malaria endemicity, the

Table 1 Source, spatial and temporal resolution of remote sensing (RS) data

\begin{tabular}{|c|c|c|c|}
\hline Predictor & $\begin{array}{c}\text { Spatial } \\
\text { Resolution }\end{array}$ & $\begin{array}{l}\text { Temporal } \\
\text { Resolution }\end{array}$ & Source \\
\hline Day land surface temperature (day LST) & $1 \times 1 \mathrm{~km}^{2}$ & 8 days & MODIS \\
\hline Night land surface temperature (night LST) & $1 \times 1 \mathrm{~km}^{2}$ & 8 days & MODIS \\
\hline Normalized difference vegetation index (NDVI) & $0.25 \times 0.25 \mathrm{~km}^{2}$ & 16 days & MODIS \\
\hline Land cover & $1 \times 1 \mathrm{~km}^{2}$ & - & MODIS \\
\hline Rainfall estimate (RFE) & $8 \times 8 \mathrm{~km}^{2}$ & daily & ADDS \\
\hline Elevation & $1 \times 1 \mathrm{~km}^{2}$ & - & USGS \\
\hline Region (urban/rural) & $1 \times 1 \mathrm{~km}^{2}$ & - & HealthMapper \\
\hline Water bodies (rivers, lakes \& wetlands) & $1 \times 1 \mathrm{~km}^{2}$ & - & HealthMapper \\
\hline Population counts & $0.5 \times 0.5 \mathrm{~km}^{2}$ & - & Landscan2006 \\
\hline
\end{tabular}


duration of the malaria transmission season and environmental factors, there is an elapsing (lag) time between the climatic suitability for malaria transmission and the occurrence of the disease. To determine this period, which may differ among environmental factors, a lag time analysis was carried out. A lag time is defined as a period prior to the survey during which an average value of the climatic factor was calculated. However, the first 16 days preceding the survey were excluded because parasite development in the mosquito takes around two weeks before the mosquito becomes infectious. For each climatic predictor, a number of analyses variables were created. These variables represent short and long term mean averages of the values of the climatic factor at different lag times. For factors extracted at 1- or 8-day temporal resolution, the lag times were multiples of 8 days (that is $8,16, \ldots, 360$ ). For NDVI which was extracted at 16 days temporal resolution, the lag times were multiples of 16 days up to one year. At the end 45 lag time variables were created for rainfall, day and night LST and 22 lag time variables for NDVI.

Bivariate logistic regression models were fitted to assess the relation between the parasitaemia risk outcome and the environmental lag time variables. For each climatic factor the lag time variable, which was further considered in the analysis, was the one giving a model with the smallest Akaike's Information Criterion (AIC). All covariates which were significant in the bivariate analysis at $15 \%$ significance level, determined by likelihood ratio tests, were included in a multiple geostatistical logistic regression analysis.

Several geostatistical multiple logistic regression models were fitted to assess and capture potential non-linearity in the malaria-environment relation. These models included covariates (i) in continuous scales (ii) in categorical scales with categories based on quartiles and (iii) fitted by penalized and basis spline ( $\mathrm{P}$ - and B-splines) curves (see Additional file 1). The model with the best predictive ability was chosen via a model validation procedure. In the geostatistical model specification, spatial correlation was taken into account by including household location-specific random effects and assuming that they derive from a multivariate Gaussian spatial process with zero mean [8]. The covariance between any pair of locations was assumed to be an exponential function of distance between the locations. Covariates and random effects were modelled on the logit scale of the parasitaemia risk parameters. The above geostatistical models have at least as many parameters as the number of locations, but model fit is possible via MCMC simulation methods. Exploratory analyses suggested weak spatial correlation therefore non-spatial models (having smaller numbers of parameters) were also fitted. The model with the best predictive ability was employed to predict the risk at the unsampled locations via Bayesian kriging. Predictions were made over a grid of around 100,000 pixels to obtain a parasitaemia risk map for Zambia.

\section{Model fit and validation}

A random sample of 89 (training) locations was selected for model fit, and the predictive ability of the models was assessed on the remaining 20 (test) locations. The range of distances from the selected test locations to the nearest training location varies from $900 \mathrm{~m}$ to $76 \mathrm{~km}$, with a median of about $26 \mathrm{~km}$, suggesting that the locations are representative of the underlying spatial process. Model predictions were compared using the following three approaches $[16,17]:$ i) the model with the highest proportion of test locations falling within the 95\% Bayesian confidence (credible) interval (CI) and ii) distance measures between observed and predicted parasitaemia prevalence calculated by the Kullback-Leibler (KL) divergence and an analogue to the $\chi^{2}$-test. In particular, for each test location the posterior predictive distribution (PPD) was computed using each fitted model. Based on the PPD, Bayesian confidence intervals were calculated with probability coverage of $95 \%$. Each credible interval was examined weather the test locations were falling within that interval. The model predicting the largest number of test locations within the $95 \%$ CI of smallest width was considered as the best one. The KL calculates the mean divergences between the observed and predicted parasitaemia prevalence on the logit scales weighted by the observed value. The analogue to the $\chi^{2}$-test is based on the squared distance between the median of the PPD and the observed parasitaemia prevalence divided by the observed prevalence. The model giving the smallest divergence or the smallest distance is considered as the best model.

A mathematical description of the models used is given in Additional file 1. The statistical analysis was carried out in Stata/SE 9.2 (StataCorp LP), Winbugs (Imperial College and Medical Research Council, UK) and in specialized software written by the authors in Fortan 95 (Digital Equipment Corporation) programming language using standard numerical libraries (Numerical Algorithms Group Ltd).

\section{Results}

The MIS included 2364 children under five years of age, randomly sampled over 120 cluster locations. However, a sample of only 1324 children at 109 cluster locations had complete parasitological data linked to a geo-located household in order to estimate the distribution of parasitaemia risk. The study profile is given in Figure 1 . The sample locations with the observed parasitaemia prevalence are shown Figure 2. 


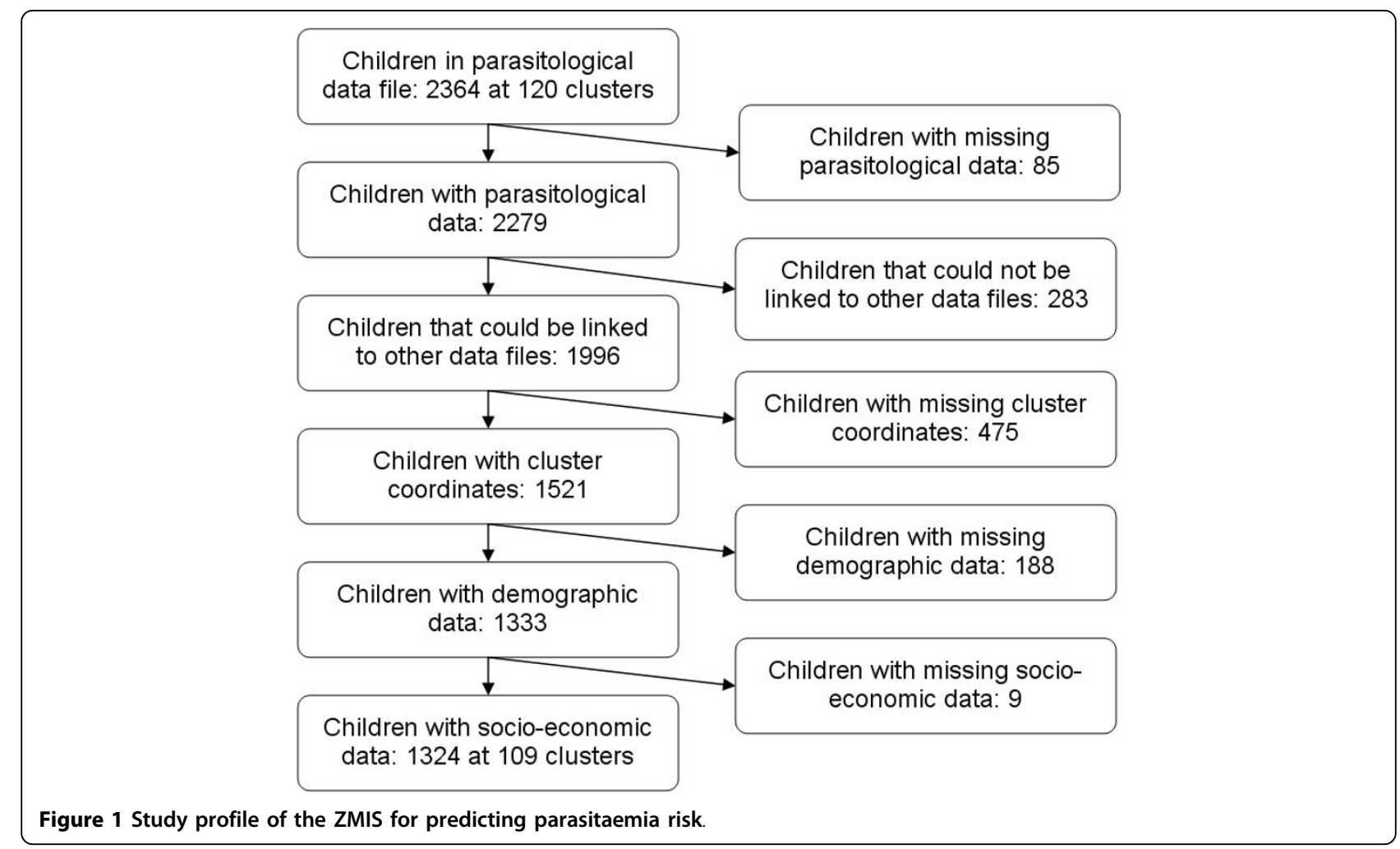

The lag time analysis suggested that the following periods preceding the survey are best (in terms of model fit) for summarizing the climatic factors: 2.7 months for rainfall, 1 month for NDVI, 1.5 months for day and night LST. The geographical distributions of the environmental factors summarized at the above lag times are displayed in Figure 3.

Non-spatial bivariate logistic regression analyses and the likelihood ratio test indicated that all RS factors were significant at $15 \%$ significance level (results not presented). All these variables were further included in the geostatistical analysis. Exploratory analysis indicated non-linearity in the relation between the parasitaemia risk and the following environmental predictors: NDVI, rainfall, day and night LST. Various Bayesian multiple logistical regression models (spatial as well as non-spatial) were fitted modelling the non-linearity of the above factors via spline curves or categorical covariates. In

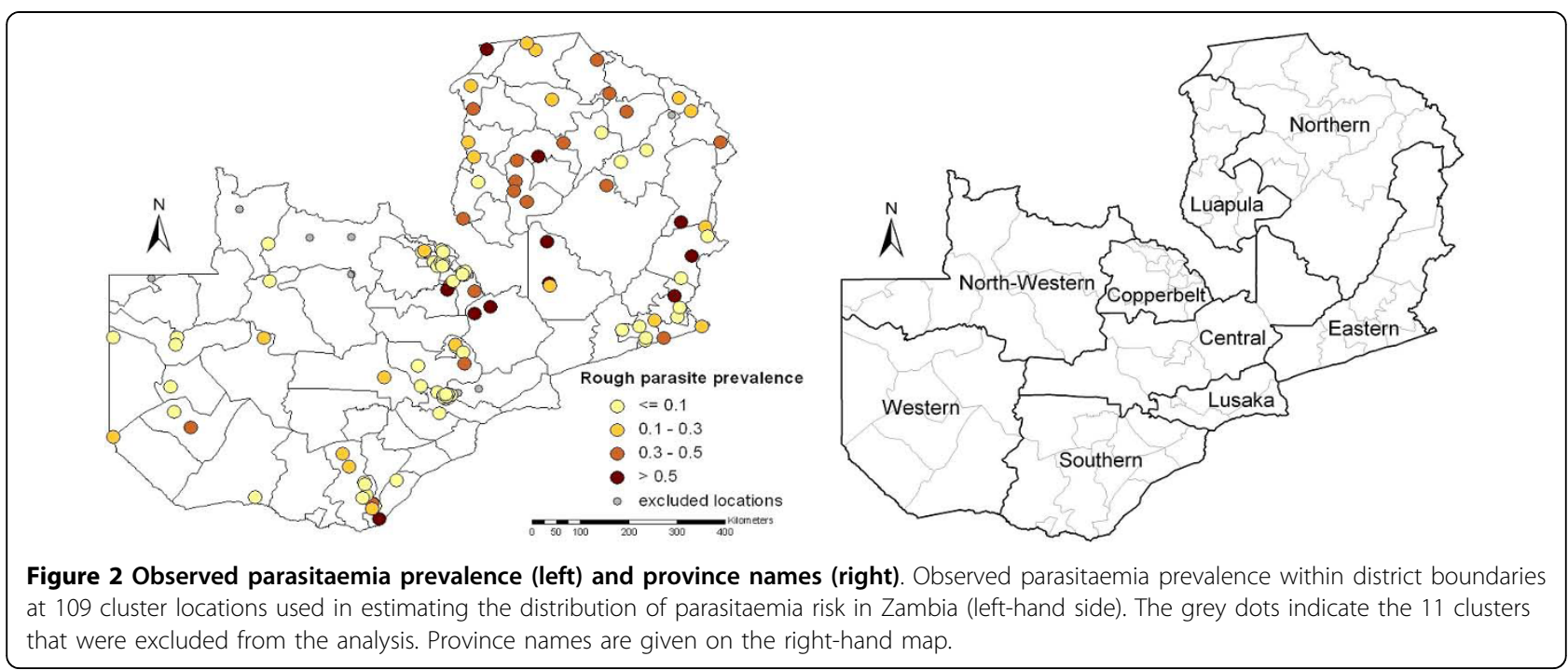




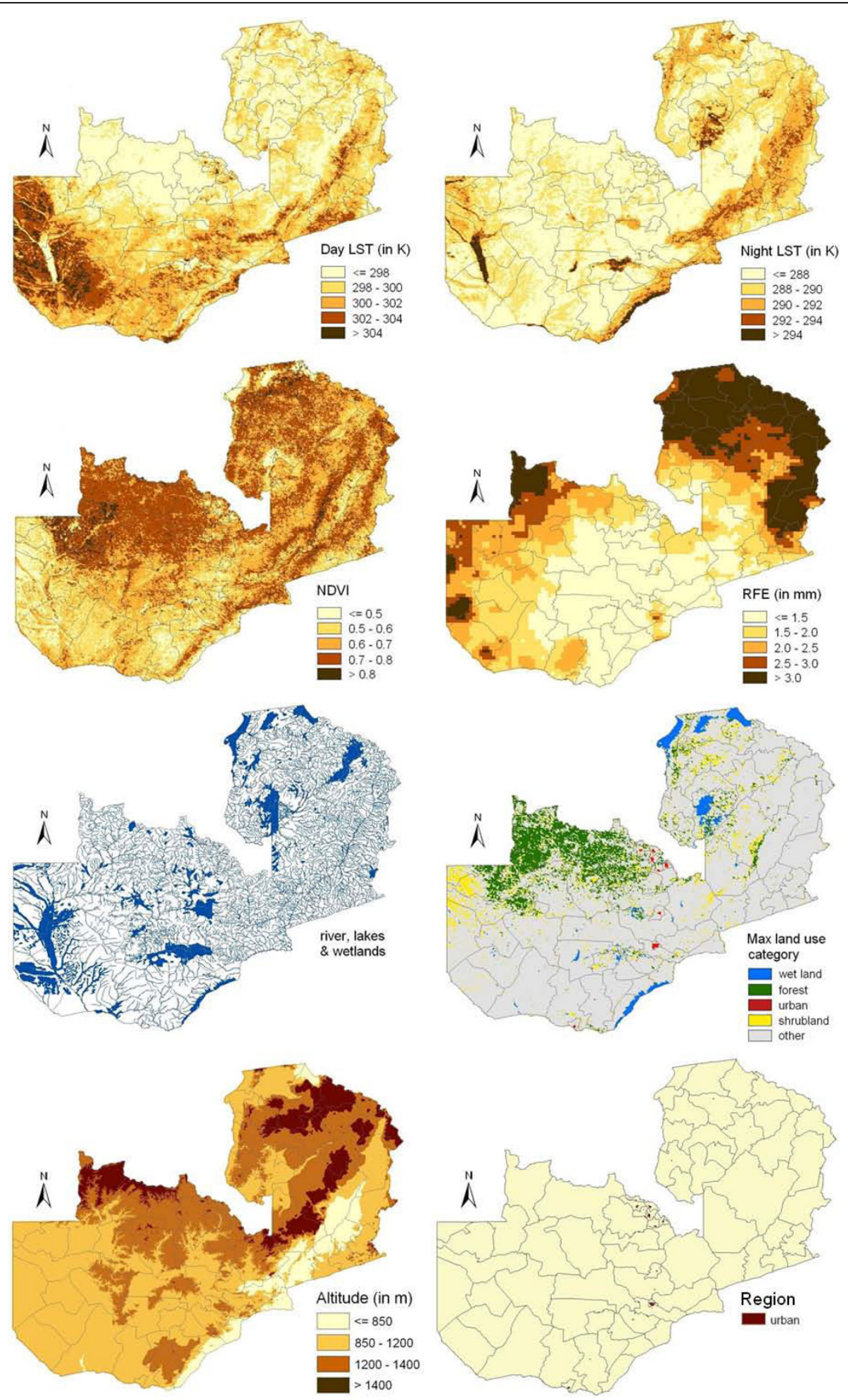

Figure 3 Spatial distribution of remotely sensed covariates in Zambia. The climatic factors were summarized over a period preceding the survey indicated by the lag time analysis (day LST, night LST, NDVI, rainfall). The land use map presents the most frequent land use category in a buffer of $3 \mathrm{~km}$ around every pixel. 
Table 2 Model validation summary for the spatial (s) and non-spatial (ns) models

\begin{tabular}{lccc}
\hline Model & Cl (width) & KL & $\chi^{2}$ \\
\hline Linear (ns) & $60 \%(0.58)$ & 21.49 & 4867 \\
Linear (s) & $50 \%(0.59)$ & 22.58 & 4459 \\
Categorical (ns) & $60 \%(0.69)$ & 30.81 & 6943 \\
Categorical (s) & $60 \%(0.68)$ & 30.33 & 6143 \\
P-spline (ns) & $60 \%(0.61)$ & 23.11 & 8194 \\
P-spline (s) & $65 \%(0.61)$ & 22.29 & 7417 \\
B-spline (ns) & $70 \%(0.72)$ & 27.59 & 26698 \\
B-spline (s) & $70 \%(0.72)$ & 28.85 & 26846
\end{tabular}

Comparison of Bayesian credible intervals (Cl) of $95 \%$ probability coverage with their corresponding width, Kullback-Leibler divergences $(\mathrm{KL})$ and the $\chi^{2}$ test analogue on 20 test locations.

addition all models included, land cover types, region type (urban/rural), altitude and distance to the nearest water bodies as categorical covariates. Results on model validation are presented in Table 2 . The B-spline models were able to predict correctly most of the test locations (70\%) within the 95\% CI however the B-spline models produced always $95 \%$ CIs with largest absolute widths. In comparison, the spatial P-spline model is able to predict correctly nearly the same proportion of test locations $(65 \%)$ within a $95 \% \mathrm{CI}$ with considerably smaller width. Among those models predicting $60 \%$ of test locations correctly within a $95 \% \mathrm{CI}$, the non-spatial model with the linear terms had the smallest absolute width. This model shows additionally the smallest KL divergence, followed by the spatial P-spline model, and the second smallest $\chi^{2}$-value. The spatial model with linear terms has the smallest $\chi^{2}$-value. Based on model validation results the non-spatial model with the linear terms was chosen as the final model used for prediction, due to the very good KL divergence and $\chi^{2}$-test results and the smallest width of the CIs. This model has also the advantage of a small number of model covariates (compared to the second best model based on spatial Psplines) avoiding over-parameterization problems. The model was employed to predict the parasitaemia risk at unsampled locations and included the following predictors: proportion of each land cover type (excluding the "other" category) within a $3 \mathrm{~km}$ radius around the location, categorical covariates for the environmental predictors (altitude, region type and distance to the nearest water bodies) and linear climatic predictors (NDVI, rainfall, day and night LST).

Predictions obtained at around 100,000 pixels via Bayesian kriging are shown in Figure 4. The predicted parasitaemia prevalence is ranging between $0.8 \%$ and $80.9 \%$ based on the posterior predictive distribution, while the observed data vary between $0.2 \%$ and $59.8 \%$. The overall prevalence (mean of the prediction for every pixel) is approximately $26.4 \%$ with a standard deviation of $15.2 \%$ (observed locations: $22.8 \%$ with $16.4 \%$ standard deviation). Relatively low risks areas $(<10 \%)$ were frequently predicted for North-Western, Western and Southern province in comparison to the high risk areas $(>50 \%)$ mainly found in Eastern province and adjacent regions. Estimates of the corresponding prediction error are depicted in Figure 5. The map shows that regions with high prediction errors have high parasitaemia risk. These are mainly areas with sparse survey locations.

The parasitaemia risk estimates were combined with the estimated number of children below five years living in the areas of the corresponding pixels to calculate the number of children with parasitaemia. These estimates are presented in Figure 6 in map form and in Table 3 as total counts at province level. Taking into account the population distribution, the average prevalence of parasitaemia risk is approximately $20.0 \%$. Lusaka province has the lowest population-adjusted prevalence level of $7.3 \%$ and is the only province with a predicted mean prevalence of $<10 \%$. Northern province has the highest population-adjusted prevalence of $38.3 \%$ followed by Luapula (30.3\%) and Eastern province (27.7\%).

To assess the effects of malaria interventions in Zambia after adjusting for climatic and environmental effects, the above model was fitted again with three additional covariates: socio-economic status of the household, indoor residual spraying within the last 12 months and presence of at least one bed net in the household. These covariates were not used for prediction as accurate estimates of their distribution in Zambia do not exist for 2006. The regression coefficients of this model (multivariate non-spatial) are given in Table 4 together with the bivariate non-spatial logistic regression models. In addition, results of the non-spatial model with linear terms and the second best model (spatial P-spline) are presented. The results of the bivariate regression models reflect significant negative relations with day LST, proportion of urban areas within a $3 \mathrm{~km}$ buffer, the region type with urban areas having lower parasitaemia risk, distance to the nearest water body, altitude levels above $1.4 \mathrm{~km}$, socio-economic status $\left(4^{\text {th }}\right.$ and $5^{\text {th }}$ quintile), spraying and presence of bed nets. Positive significant relations were detected with night LST, NDVI, rainfall within the last 2.7 months and proportion of wetlands in the surrounding area. The implementation of the multivariate non-spatial model indicated a loss of significant covariates. The only remaining significant parameter was the presence of at least one bed net in a household which reduces the odds of parasitaemia in children by $40 \%$ (CI: $12 \%, 61 \%$ ). The final prediction model (non-spatial model with the linear terms) without the additional household specific covariates showed no significant correlation between the predictors and the parasitaemia risk. The non-spatial 


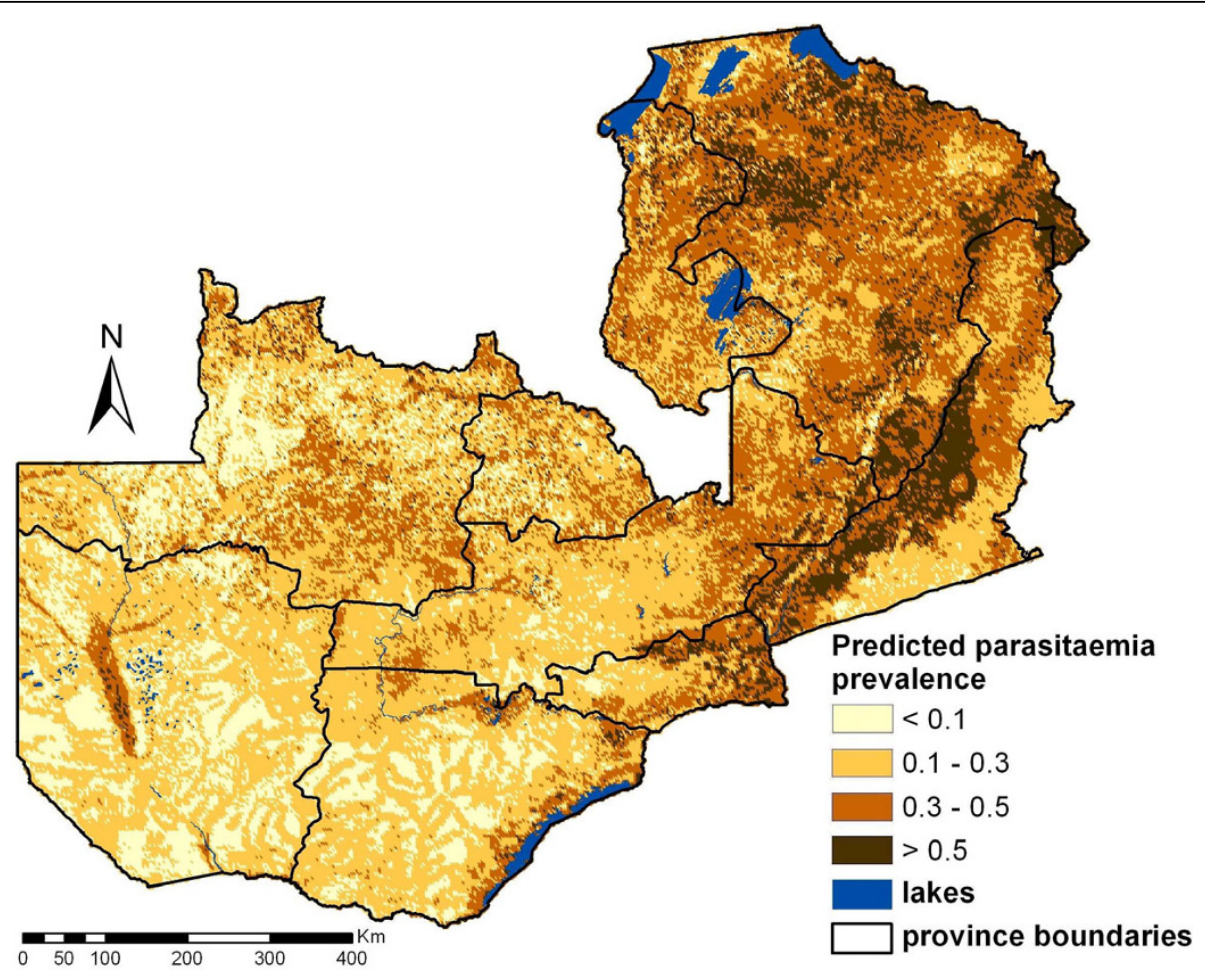

Figure 4 Predicted parasitaemia risk map for children $<5$ years in Zambia. The map is based on a Bayesian logistic regression model with linear terms for day LST, night LST, NDVI and rainfall. The estimates correspond to the median of the posterior predictive distributions computed over 100,000 pixels.

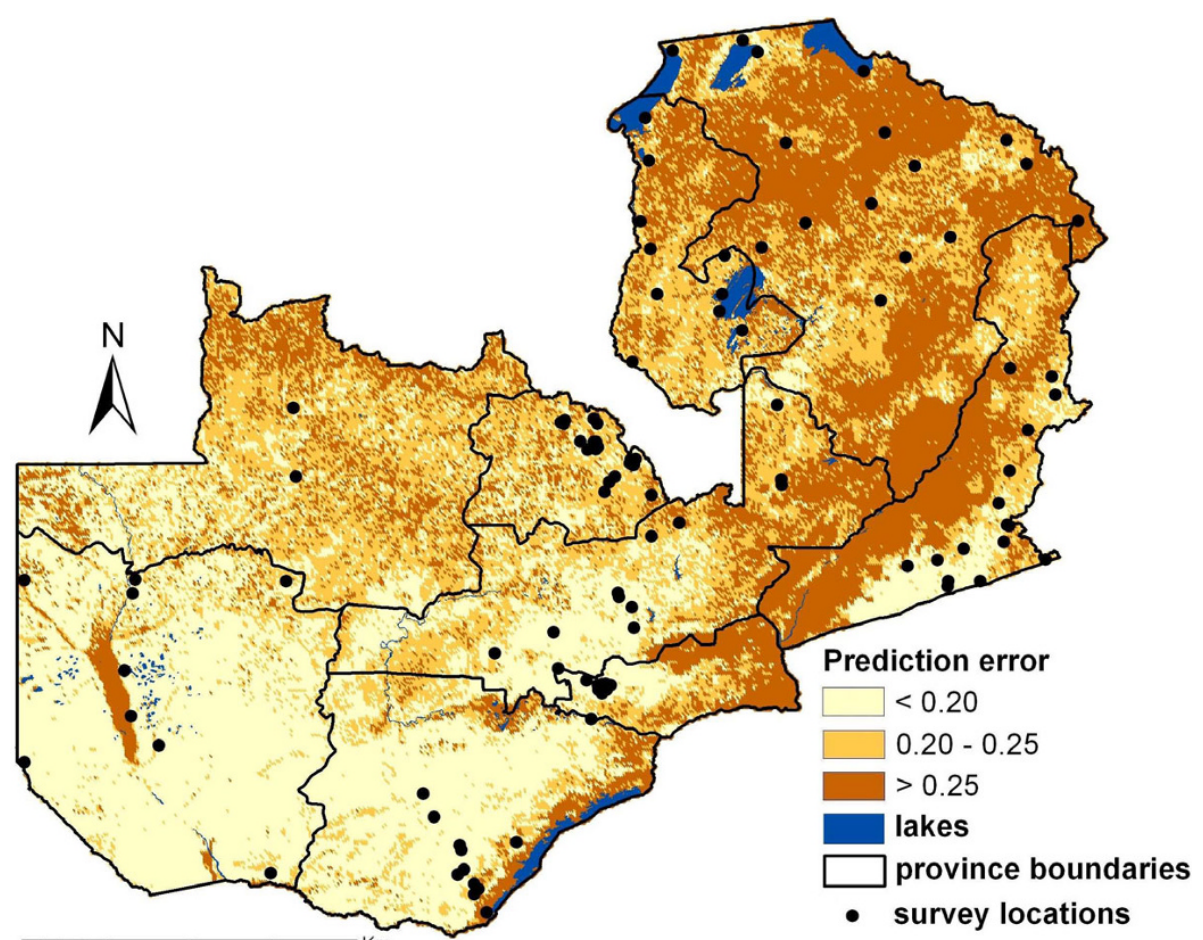

Figure 5 Prediction error of the parasitaemia risk estimates given in Figure 4 


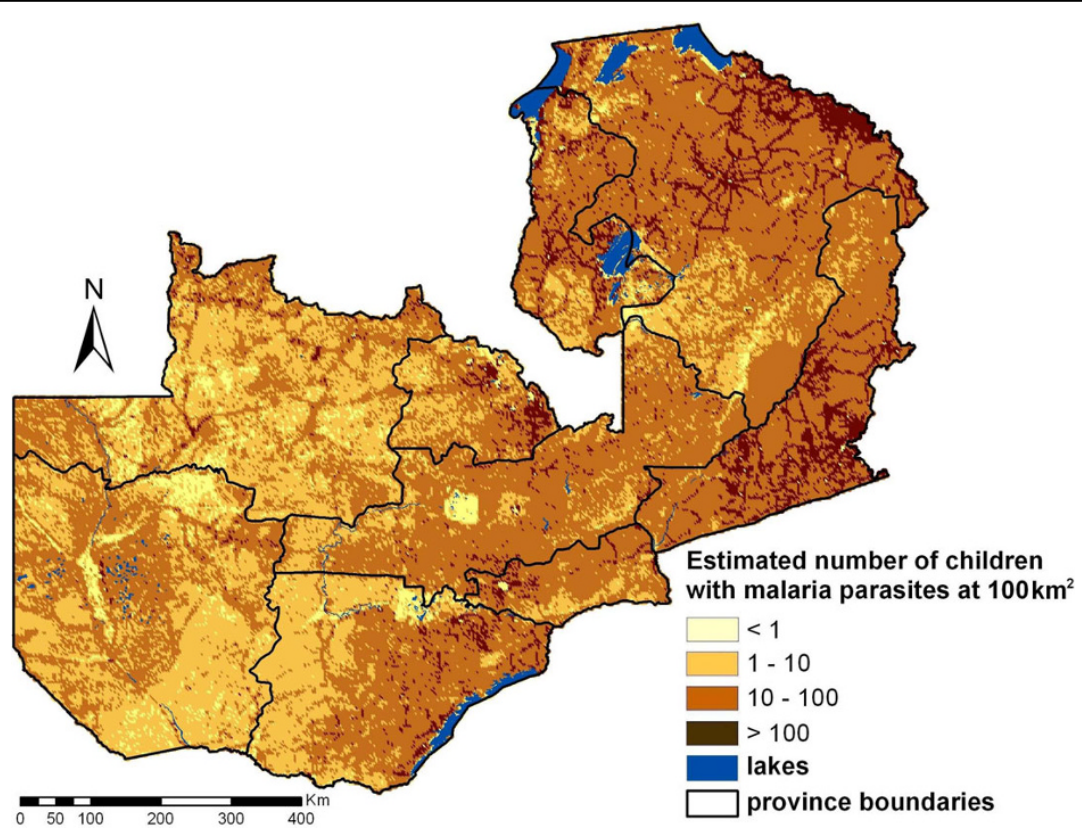

Figure 6 Estimated number of infected children $<5$ years per $100 \mathrm{~km}^{2}$.

variance was around 1.7. The P-spline spatial model estimates a ratio of spatial to the total variation of almost 1:2. The minimum distance at which the spatial correlation is lower than $5 \%$ estimated by the spatial model is 380 meters ( $95 \%$ confidence interval: $210 \mathrm{~m}$, $3,390 \mathrm{~m}$ ) which is even lower than the resolution of the grid used for prediction $(3 \mathrm{~km})$. This suggests a very weak spatial correlation and supports the choice of the non-spatial model as the one with the best predictive ability.

\section{Discussion}

The ZMIS in 2006 was the first nation-wide malaria survey which combined parasitological data with other malaria indicators such as bed net use, indoor residual spraying and household related aspects. The aim of the survey was to estimate the coverage of interventions and the malaria related burden in children less than five years. However, the MIS data are also a very important source of information for estimating parasitaemia risk at local scales and thus for identifying the high-risk areas that require high intervention coverage and continuous monitoring. Combining parasitaemia risk estimates with population data, the number of infected children can be estimated which can help for better resource allocation, health management and targeted additional interventions to achieve the highest risk reduction for the most populated areas. Repeated surveys will enable the

Table 3 Predicted number of children $<5$ years with malaria parasites in the blood (per province)

\begin{tabular}{|c|c|c|c|c|c|c|}
\hline Province & $\begin{array}{r}\text { Prev } 1 \\
\text { (in \%) } \\
\end{array}$ & $\begin{array}{l}\text { Children } \\
<5 \text { years }\end{array}$ & $\begin{array}{l}\text { Infected } \\
\text { Children }\end{array}$ & $95 \%$ & & $\begin{array}{r}\text { Prev 2 } \\
\text { (in \%) }\end{array}$ \\
\hline Central & 26.0 & 182,847 & 34,572 & 21,589 & 50,252 & 18.9 \\
\hline Copperbelt & 23.3 & 311,317 & 37,763 & 18,572 & 70,719 & 12.1 \\
\hline Eastern & 37.4 & 240,137 & 66,614 & 46,297 & 87,219 & 27.7 \\
\hline Luapula & 32.0 & 125,049 & 37,943 & 29,039 & 47,638 & 30.3 \\
\hline Lusaka & 31.8 & 275,120 & 20,134 & 8,121 & 46,849 & 7.3 \\
\hline North-Western & 21.0 & 128,935 & 29,011 & 16,200 & 51,616 & 22.5 \\
\hline Northern & 39.1 & 277,764 & 106,322 & 79,379 & 135,701 & 38.3 \\
\hline Southern & 18.8 & 243,743 & 33,430 & 19,862 & 53,854 & 13.7 \\
\hline Western & 14.4 & 147,229 & 20,321 & 12,730 & 30,232 & 13.8 \\
\hline Total & 26.4 & $1,932,141$ & 386,110 & 251,789 & 574,080 & 20.0 \\
\hline
\end{tabular}

Estimates are based on the mean and the $95 \%$ confidence intervals $(\mathrm{Cl})$ of the posterior predictive distribution of the non-spatial model with linear terms. 
Table 4 Parasitaemia risk predictors of different models

\begin{tabular}{|c|c|c|c|c|}
\hline Covariates & $\begin{array}{c}\text { Bivariate } \\
\text { non-spatial } \\
\text { OR }(95 \% \mathrm{Cl})\end{array}$ & $\begin{array}{l}\text { Multivariate } \\
\text { non-spatial } \\
\text { OR }(95 \% \mathrm{Cl})\end{array}$ & $\begin{array}{l}\text { Prediction } \\
\text { model } \\
\text { OR }(95 \% \mathrm{Cl})\end{array}$ & $\begin{array}{c}\text { Spatial P-spline model } \\
\text { OR }(95 \% \mathrm{Cl})\end{array}$ \\
\hline Day LST & $0.49(0.43,0.57)$ & $0.65(0.37,1.15)$ & $0.61(0.32,1.17)$ & $*$ \\
\hline Night LST & $1.23(1.09,1.40)$ & $1.21(0.77,1.88)$ & $1.18(0.79,1.77)$ & * \\
\hline NDVI & $2.25(1.90,2.66)$ & $1.28(0.67,2.73)$ & $1.29(0.66,2.77)$ & * \\
\hline Rainfall & $1.56(1.37,1.76)$ & $1.21(0.85,1.68)$ & $1.18(0.80,1.73)$ & * \\
\hline \multicolumn{5}{|l|}{ Land cover covariates } \\
\hline Wetland & $1.20(1.07,1.34)$ & $0.97(0.62,1.55)$ & $0.98(0.67,1.48)$ & $0.72(0.40,1.37)$ \\
\hline Forest & $0.96(0.84,1.10)$ & $0.72(0.43,1.08)$ & $0.72(0.43,1.10)$ & $0.64(0.38,0.99)$ \\
\hline Urban & $0.35(0.25,0.50)$ & $0.70(0.38,1.21)$ & $0.71(0.37,1.29)$ & $0.75(0.33,1.48)$ \\
\hline Shrubland & $1.06(0.94,1.20)$ & $1.07(0.76,1.53)$ & $1.05(0.71,1.47)$ & $1.07(0.72,1.53)$ \\
\hline \multicolumn{5}{|l|}{ Region (rural) } \\
\hline urban & $0.17(0.11,0.25)$ & $0.53(0.14,2.03)$ & $0.37(0.11,1.13)$ & $0.43(0.12,1.50)$ \\
\hline \multicolumn{5}{|c|}{ Distance to water bodies $(<1000 \mathrm{~m})$} \\
\hline $1000-2499$ & $0.71(0.53,0.96)$ & $0.73(0.29,1.86)$ & $0.71(0.29,1.72)$ & $0.55(0.19,1.50)$ \\
\hline $2500-4999$ & $0.54(0.38,0.77)$ & $0.61(0.20,1.55)$ & $0.60(0.19,1.72)$ & $0.49(0.17,1.42)$ \\
\hline$\geq 5000$ & $0.11(0.04,0.30)$ & $0.22(0.03,1.40)$ & $0.21(0.03,1.39)$ & $0.20(0.02,1.93)$ \\
\hline \multicolumn{5}{|l|}{ Altitude $(<850 \mathrm{~m})$} \\
\hline 850-1199 & $0.72(0.49,1.06)$ & $0.21(0.03,1.70)$ & $0.22(0.03,1.80)$ & $0.20(0.02,1.92)$ \\
\hline 1200-1399 & $0.73(0.49,1.09)$ & $0.32(0.03,3.27)$ & $0.30(0.03,3.29)$ & $0.27(0.02,4.81)$ \\
\hline$\geq 1400$ & $0.48(0.25,0.92)$ & $0.74(0.04,10.2)$ & $0.57(0.03,7.68)$ & $0.32(0.02,7.86)$ \\
\hline \multicolumn{5}{|l|}{$\begin{array}{l}\text { Socio-economic index } \\
\text { (1 }{ }^{\text {st }} \text { quintile) }\end{array}$} \\
\hline $2^{\text {nd }}$ quintile & $1.06(0.75,1.50)$ & $1.21(0.78,1.92)$ & - & - \\
\hline $3^{\text {rd }}$ quintile & $0.85(0.60,1.22)$ & $1.31(0.75,2.23)$ & - & - \\
\hline $4^{\text {th }}$ quintile & $0.28(0.17,0.46)$ & $0.75(0.33,1.75)$ & - & - \\
\hline $5^{\text {th }}$ quintile & $0.09(0.04,0.19)$ & $0.40(0.09,1.56)$ & - & - \\
\hline \multicolumn{5}{|l|}{ Interventions } \\
\hline IRS & $0.16(0.07,0.36)$ & $1.73(0.42,6.90)$ & - & - \\
\hline \multirow[t]{2}{*}{ Bed nets } & $0.59(0.46,0.77)$ & $0.60(0.39,0.88)$ & - & - \\
\hline & & Mean $(95 \% \mathrm{Cl})$ & Mean $(95 \% \mathrm{Cl})$ & Mean $(95 \% \mathrm{Cl})$ \\
\hline Range (in km) & - & - & - & $0.38(0.21,3.39)$ \\
\hline$\sigma^{2}$ (spatial error) & - & - & - & $0.98(0.01,2.77)$ \\
\hline$\tau^{2}$ (measurement error) & - & $1.77(0.90,3.23)$ & $1.71(0.93,2.84)$ & $0.82(0.01,2.69)$ \\
\hline
\end{tabular}

Associations between parasitaemia risk and predictors of the non-spatial model with linear terms and the Bayesian spatial logistic regression P-spline model presented as odds ratios (OR) with their respective $95 \%$ confidence intervals (Cl).

*: regression coefficients are based on P-spline curves

evaluation of the effectiveness of on-going interventions. Already the Zambian Ministry of Health and partners have completed the second ZMIS in the country in 2008 and planning another follow-up survey in 2010.

Prior to the MIS, compiled historical survey data has been used to obtain estimates of parasitaemia risk at high resolution. The mapping malaria risk in Africa (MARA) project was initiated in 1998 with the aim to compile published and unpublished malaria survey data in Africa. MARA is the most comprehensive malariometric database compiling data from 1900 up to date. The MARA data has been analysed using rigorous spatial statistical modelling [17-20] to obtain high-resolution malaria risk estimates at regional and country-level in Africa. However, risk estimates of historical data do not reflect the current malaria situation, which is influenced by on-going interventions. The surveys are not representative as high risk areas tend to be over-represented. In addition historical surveys have been conducted between various locations using different methodologies, including different age groups and carried out at different seasons. On the contrary, MISs do not suffer from these drawbacks. The MIS locations are randomly chosen and the data are available at individual level allowing for estimation of age-specific risk. Another advantage of the MIS data is the household level information available which gives the possibility of differentiating the contribution of climate, socio- 
economic characteristics and control interventions to the overall parasitaemia risk. If in addition these data are known at high spatial resolution, they can be included in the geostatistical model to obtain more accurate predictions of the malaria risk.

This study created the first contemporary empirical parasitaemia risk map for Zambia. Many existing maps on malaria transmission rely only on rough geographical and climatic iso-lines and expert opinions. Until 1998, none of the maps had a numerical definition, hence the malaria risk maps were not comparable and trustworthy. Then, Hay et al [21] produced a climatic map for malaria transmission in Kenya followed by Craig et al [22] who developed a climatic suitability malaria risk map for the whole Africa. Up to now there are only a few maps containing also empirical data, the first map of this kind was published in 2000 for Mali by Kleinschmidt et al [18]. For Zambia, the first empirical malaria risk map was produced by Hay et al [23] and is part of a global risk map based on historical data.

Malaria survey data are expected to be correlated in space. Spatial correlation at short distances is introduced by the transmission process driven by the flight range of the mosquito vector while at wider ranges spatial correlation reflects common exposures to environmental conditions, which influence mosquito survival and longevity. High spatial resolution risk estimation requires prediction at locations where malaria survey data are not available. High-resolution environmental data can be obtained via remote sensing. GIS software has excellent mapping capabilities and it is a very useful tool for processing RS data.

Statistical techniques model the relation between parasitaemia risk and risk factors (environmental, possible interventions, socio-economic factors) via a logistic regression model, which is further used for prediction. Standard statistical methods assume independence of the survey locations. Violating this assumption, when modelling spatially-correlated malaria survey data may lead to imprecise estimates of the risk, the significance of the risk factors and of the prediction error. Similarly, modelling spatial correlation in weakly correlated data increases the number of model parameters and decreases the precision of the estimates. Geostatistical models take into account spatial correlation by introducing an additional parameter (random effect) at each survey location and assume that geographical dependence is a function of distance between locations. Depending on the number of survey locations these models can be highly parameterized and they can only be estimated using Bayesian inference and MCMC simulation. Bayesian geostatistical models have been employed in malaria risk estimation by e.g. Diggle et al [19], Gemperli et al [20,24] and Gosoniu et al [16,17].
However, in this study the Bayesian geostatistical model has estimated very low spatial correlation dropping to less than $5 \%$ at distances larger than $380 \mathrm{~m}$, which is lower than the spatial resolution of the pixel size used for prediction ( $3 \mathrm{~km}$ by $3 \mathrm{~km}$ ). The non-spatial model was superior to the fitted spatial models because spatial correlation is only present at a very local scale indicated by the flight range of the mosquito rather than environmental covariates. Possible reasons might be on-going interventions, which determine mosquito densities and parasitaemia and therefore reduce the influence of environmental predictors on the mosquito.

Previous models of malaria transmission addressed non-linearity between the risk of the malaria-related outcome (in the logit scale) and its predictor solely by categorizing the non-linear covariates. In this study, non-linearity is modelled additionally by using different types of spline curves. The resulting risk estimates suggested that predictions are sensitive to the model fitted. However, model validation indicated that models based on linear terms are superior to non-linear models in Zambia. Even though the P-spline model had the higher predictive ability at $95 \%$ CIs, the linear model was considered as the one with the best predictive ability due to the smaller range of these intervals and the results of the KL divergence and $\chi^{2}$-measures between observedpredicted prevalence data. This model has also the advantage of an easy interpretability of the regression coefficients for non-statisticians in comparison to the spline curve ones.

None of the regression coefficients of the final prediction model were significant. However, they are needed to determine the mean risk estimate for each location and excluding them reduces heavily the model predictive ability (results have not been shown). The lack of significance of the environmental factors is partially explained by the effects of malaria interventions, which can have stronger influence on the parasitaemia risk than the environmental factors. In fact, the spatial model, which adjusted for different types of interventions, indicated a significant effect of the presence of at least one bed net in a household in reducing parasitaemia risk. Therefore interventions are a major driver of parasitaemia risk in Zambia and including these data in the prediction model would increase the accuracy of model-based risk predictions. Unfortunately, in this study, the parasitaemia risk could not be predicted conditional on bed net coverage because the geographical distribution of bed net coverage was not known for the time of the ZMIS 2006. Intervention data are needed over the entire study area in order to be used for prediction purposes. Since e.g. MACEPA is putting a lot of efforts in scaling up malaria interventions in Zambia, the role of interventions is likely to even increase within 
the next couple of years. Therefore, compilation of intervention coverage data at high spatial resolution is becoming essential to create reliable risk maps.

The prediction map indicates high variation of parasitaemia risk over the country. In particular, high risk is predicted a stripe from south to east of Zambia which is characterized mainly by low altitude, high NDVI, high day and night LST. The relatively small risk in the southwest of the country might be influenced by low vegetation and rainfall while the small risk in the north-west regions could be due to low day LST and high proportion of forests. It is also possible that malaria interventions have been focused on selected areas like the Zambezi river reducing the parasite prevalence in those areas. It is interesting to note that the maps have been shown to local experts who confirmed the depicted risk patterns. The map of the prediction error of the Bayesian model could assist in improving precision of the parasitaemia risk prediction by identifying the areas of high uncertainty where additional survey locations could be randomly distributed in following MIS's and hence reduce the error and raise the precision of following studies.

The reduced smoothness of the map might be explained by the weak spatial correlation which most likely indicate that interventions counteract the environmental effects. The sampling framework of the MIS may also tend to underestimate spatial correlation because the survey clusters are partially driven by population density, where transmission is largely influenced by interventions. However the drastic changes within small distances are highlighting the importance of the highresolution maps. If prediction would have been done at smaller resolution some of the high risk areas would disappear and the burden would have been underestimated in these regions. For other purposes, which do not need such high precision, reducing the resolution can be easily done by combining neighbouring pixels.

The average predicted parasitaemia risk over the whole country was $26.4 \%$, however after adjusting for the population size the risk dropped down to $20.0 \%$. It is striking that although the average risk in Lusaka province is the forth highest of all provinces (31.8\%), after taking into account the population density the risk reduces to $7.3 \%$ indicating that the highest risk areas are the less populated. In fact Lusaka is the province of the lowest population-adjusted risk. The provinces of Copperbelt, Southern and Western have low populationadjusted risks (less than 15\%). On the other hand Northern province has the highest population-adjusted parasitaemia risk followed by Eastern province and Luapula. In addition, Northern province has the highest number of infected children less than five years old. Therefore, interventions should be concentrated in the Northern province especially at the north-eastern border and in the middle of the province. Further interventions in Copperbelt and Southern might also have a strong impact in reducing the overall burden since the total number of infected children is as high as in Luapula even though their prevalence is lower than $15 \%$.

\section{Conclusions}

The map of parasitaemia risk together with the prediction error and the population at risk give an important overview of the malaria situation in Zambia. The maps can be used by decision-makers to allocate resources and interventions to reach the most persons in the regions of highest risk to reduce the burden of malaria significantly for Zambia. The ZMIS of 2008 and 2010 will provide very important information on the changes of the parasitaemia risk over space and time and help in the evaluation of the progress of new and established intervention programmes adjusted for environmental drivers of the transmission risk.

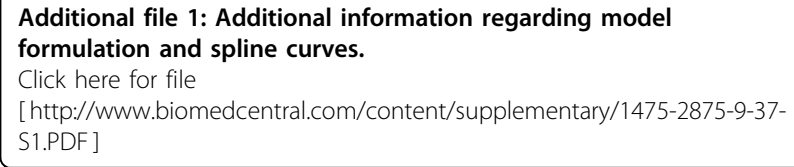

\section{Acknowledgements}

This investigation received financial support from the Swiss National Science Foundation (SNF) (Project No. 325200-118379) and the Bill and Melinda Gates Foundation (Project No. 39777). Special thanks to Prof. Thomas Smith for his valuable inputs on data analysis aspects.

\section{Author details}

'Department of Public Health and Epidemiology, Swiss Tropical Institute, PO Box, 4002 Basel, Switzerland. ${ }^{2}$ Malaria Control and Evaluation Partnership in Africa, PATH, Ferney, France. ${ }^{3}$ Departement of Public Health and Research, Ministry of Health, Zambia.

\section{Authors' contributions}

NR analysed the data and drafted the manuscript. PV was responsible for conception and design of the analysis, supervised the implementation and revised the manuscript. JMM participated in designing and coordinating the fieldwork and provided important intellectual content to this study. LG contributed in the analysis and helped to draft the manuscript. ECK gave intellectual content and revised the draft critically. VM enabled the MIS 2006 to occur and has been instrumental in championing the use of this data for guiding decisions. RWS coordinated the fieldwork and reviewed the manuscript. All authors read and approved the final manuscript.

\section{Competing interests}

The authors declare that they have no competing interests.

Received: 10 November 2009

Accepted: 1 February 2010 Published: 1 February 2010

\section{References}

1. Ministry of Health: 2008 National malaria control action plan: actions for scale-up for impact on malaria in Zambia. Zambia 2008. [http://www.nmcc. org.zm/publications.htm].

2. Ministry of Health: A road map for impact on malaria in Zambia 2006-2010: a 5-year strategic plan. Zambia 2006. [http://www.nmcc.org.zm/publications. htm]. 
3. Campbell K, Terry D, Wood S: Scaling up for impact: a model for malaria control. Zambia 2007. [http://www.path.org/files/MACEPA_Bro_2007-08-06. pdf].

4. Ministry of Health: 2009 National malaria control action plan: actions for scale-up for impact on malaria in Zambia. Zambia 2009

5. Ministry of Health, Zambia Central Statistical Office, PATH, MACEPA, CDC, University of Zambia, WHO: Zambia National Malaria Indicator Survey 2006. Zambia 2006. [http://www.nmcc.org.zm/publications.htm].

6. Craig MH, Snow RW, Le Sueur D: A climate-based distribution model of malaria transmission in sub-Saharan Africa. Parasitol Today 1999, 15:105-111.

7. Guerra CA, Gikandi PW, Tatem AJ, Noor AM, Smith DL, Hay SI, Snow RW: The limits and intensity of Plasmodium falciparum transmission: implications for malaria control and elimination worldwide. PLoS Med 2008, 5:e38.

8. Diggle PJ, Tawn JA, Moyeed RA: Model-based geostatistics. App/ Stat 1998, 47:299-350.

9. Filmer $D$, Pritchett $L H:$ Estimating wealth effects without expenditure data - or tears: an application to educational enrolments in states of India. Demography 2001, 38:115-32.

10. U.S. Geological Survey (USGS) Land Processes Distributed Active Archive Center (LP DAAC) MODIS land products. [https://pdaac.usgs.gov//pdaac/ products/modis_products_table].

11. U.S. Geological Survey (USGS) Famine Early Warning Systems Network (FEWS NET) African Data Dissemination Service (ADDS). [http:// earlywarning.usgs.gov/adds/].

12. U.S. Geological Survey (USGS) Digital Elevation Models (DEM). [http:// edc2.usgs.gov/geodata/index.php].

13. World Health Organization (WHO) The HealthMapper Database. [http:// www.who.int/health_mapping/tools/healthmapper/en/index.html].

14. Oak Ridge National Laboratory (Oak Ridge, TN) LandS $\operatorname{can}^{\mathrm{TM}}$ Global Population Database. http://www.ornl.gov/landscan/.

15. U.S. Census Bureau International Data Base (IDB) Population Division Zambia 2006. http://www.census.gov/ipc/www/idb/country.php.

16. Gosoniu L, Vounatsou P, Sogoba N, Smith T: Bayesian modelling of geostatistical malaria risk data. Geospat Health 2006, 1:127-139.

17. Gosoniu L, Vounatsou P, Sogoba N, Maire N, Smith T: Mapping malaria risk in West Africa using a Bayesian nonparametric non-stationary model. Comput Stat Data Anal 2009, 53:3358-3371.

18. Kleinschmidt I, Bagayoko M, Clarke GPY, Craig M, le Sueur D: A spatial statistical approach to malaria mapping. Int J Epidemiol 2000, 29:355-361.

19. Diggle P, Moyeed R, Rowlingson B, Thomson M: Childhood malaria in the Gambia: a case-study in model-based geostatistics. Appl Stat 2002, 51:493-506.

20. Gemperli A, Sogoba N, Fondjo E, Mabaso M, Bagayako M, Briët OJT, Anderegg D, Liebe J, Smith T: Mapping malaria transmission in West and Central Africa. Trop Med Int Health 2006, 11:1032-46.

21. Hay SI, Snow RW, Rogers DJ: Predicting malaria seasons in Kenya using multitemporal meteorological satellite sensor data. Trans $R$ Soc Trop Med Hyg 1998, 92:12-20.

22. Craig MH, Snow RW, le Sueur D: A climate-based distribution model of malaria transmission in sub-Saharan Africa. Parasitol Today 1999 15:105-111.

23. Hay SI, Guerra CA, Gething PW, Patil AP, Tatem AJ, Noor AM, Kabaria CW, Manh BH, Elyazar IRF, Brooker S, Smith DL, Moyeed RA, Snow RW: A world malaria map: Plasmodium falciparum endemicity in 2007. PLoS Med 2009, 6:e1000048.

24. Gemperli A, Vounatsou P, Kleinschmidt I, Bagayoko M, Lengeler C, Smith T: Spatial patterns of infant mortality in Mali: the effect of malaria endemicity. Am J Epidemiol 2004, 159:64-72.

doi:10.1186/1475-2875-9-37

Cite this article as: Riedel et al: Geographical patterns and predictors of malaria risk in Zambia: Bayesian geostatistical modelling of the 2006 Zambia national malaria indicator survey (ZMIS). Malaria Journal 2010 9:37.

\section{Submit your next manuscript to BioMed Central and take full advantage of:}

- Convenient online submission

- Thorough peer review

- No space constraints or color figure charges

- Immediate publication on acceptance

- Inclusion in PubMed, CAS, Scopus and Google Scholar

- Research which is freely available for redistribution

Submit your manuscript at www.biomedcentral.com/submit
Ciomed Central 